Document downloaded from:

http://hdl.handle.net/10251/56764

This paper must be cited as:

Sans, M.; Selga, J.; Rodríguez Pérez, AM.; Bonache, J.; Boria Esbert, VE.; Martín, F. (2014). Design of planar wideband bandpass filters from specifications using a two-step aggressive space mapping (ASM) optimization algorithm. IEEE Transactions on Microwave Theory and Techniques. 62(12):3341-3350. doi:10.1109/TMTT.2014.2365477

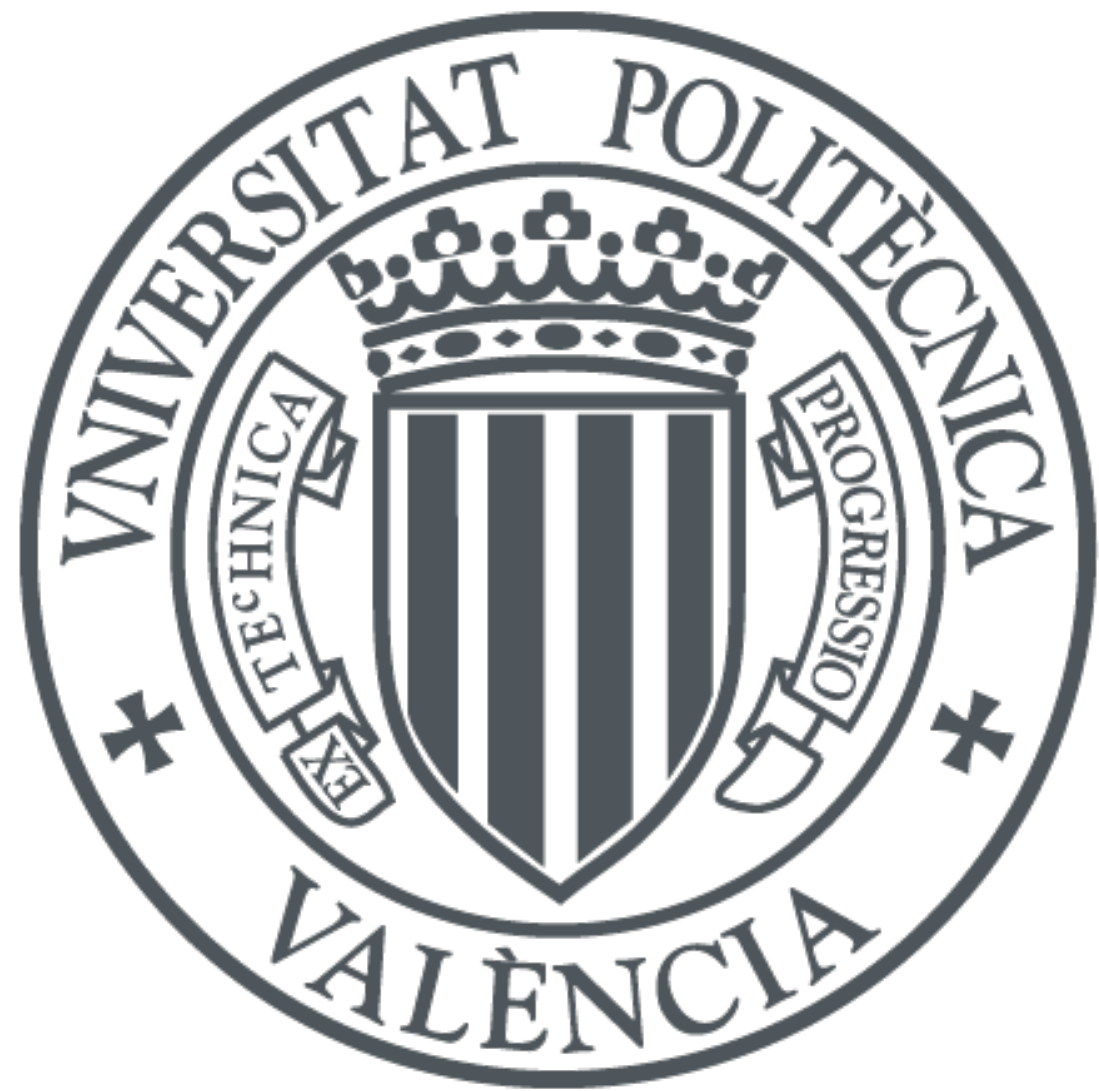

The final publication is available at

http://dx.doi.org/10.1109/TMTT.2014.2365477

Copyright Institute of Electrical and Electronics Engineers (IEEE)

Additional Information 
Document downloaded from:

http://hdl.handle.net/10251/56764

This paper must be cited as:

Sans, M.; Selga, J.; Rodríguez Pérez, AM.; Bonache, J.; Boria Esbert, VE.; Martín, F. (2014). Design of planar wideband bandpass filters from specifications using a two-step aggressive space mapping (ASM) optimization algorithm. IEEE Transactions on Microwave Theory and Techniques. 62(12):3341-3350. doi:10.1109/TMTT.2014.2365477

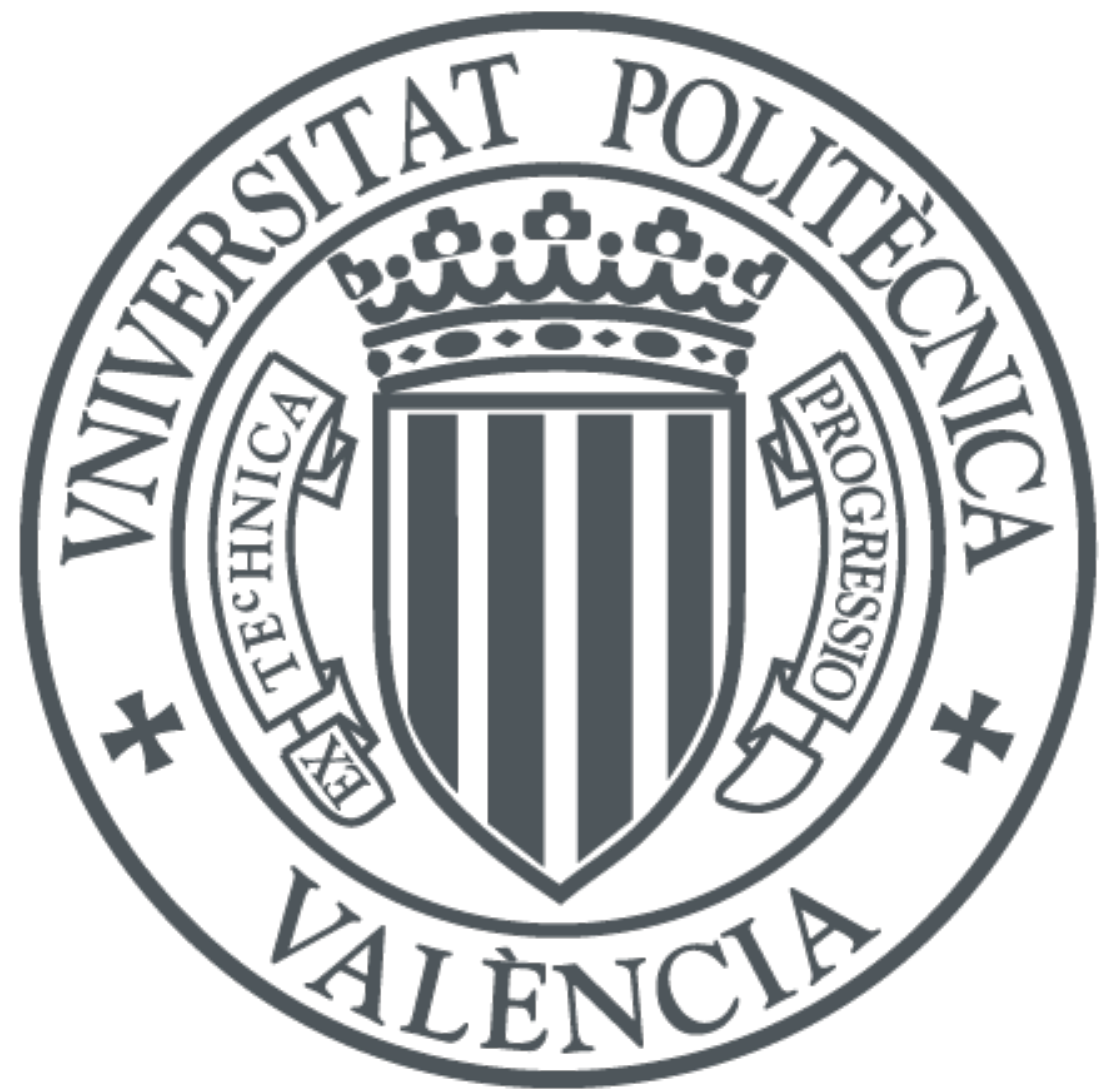

The final publication is available at

http://dx.doi.org/10.1109/TMTT.2014.2365477

Copyright Institute of Electrical and Electronics Engineers (IEEE)

Additional Information 
Document downloaded from:

http://hdl.handle.net/10251/56764

This paper must be cited as:

Sans, M.; Selga, J.; Rodríguez Pérez, AM.; Bonache, J.; Boria Esbert, VE.; Martín, F. (2014). Design of planar wideband bandpass filters from specifications using a two-step aggressive space mapping (ASM) optimization algorithm. IEEE Transactions on Microwave Theory and Techniques. 62(12):3341-3350. doi:10.1109/TMTT.2014.2365477

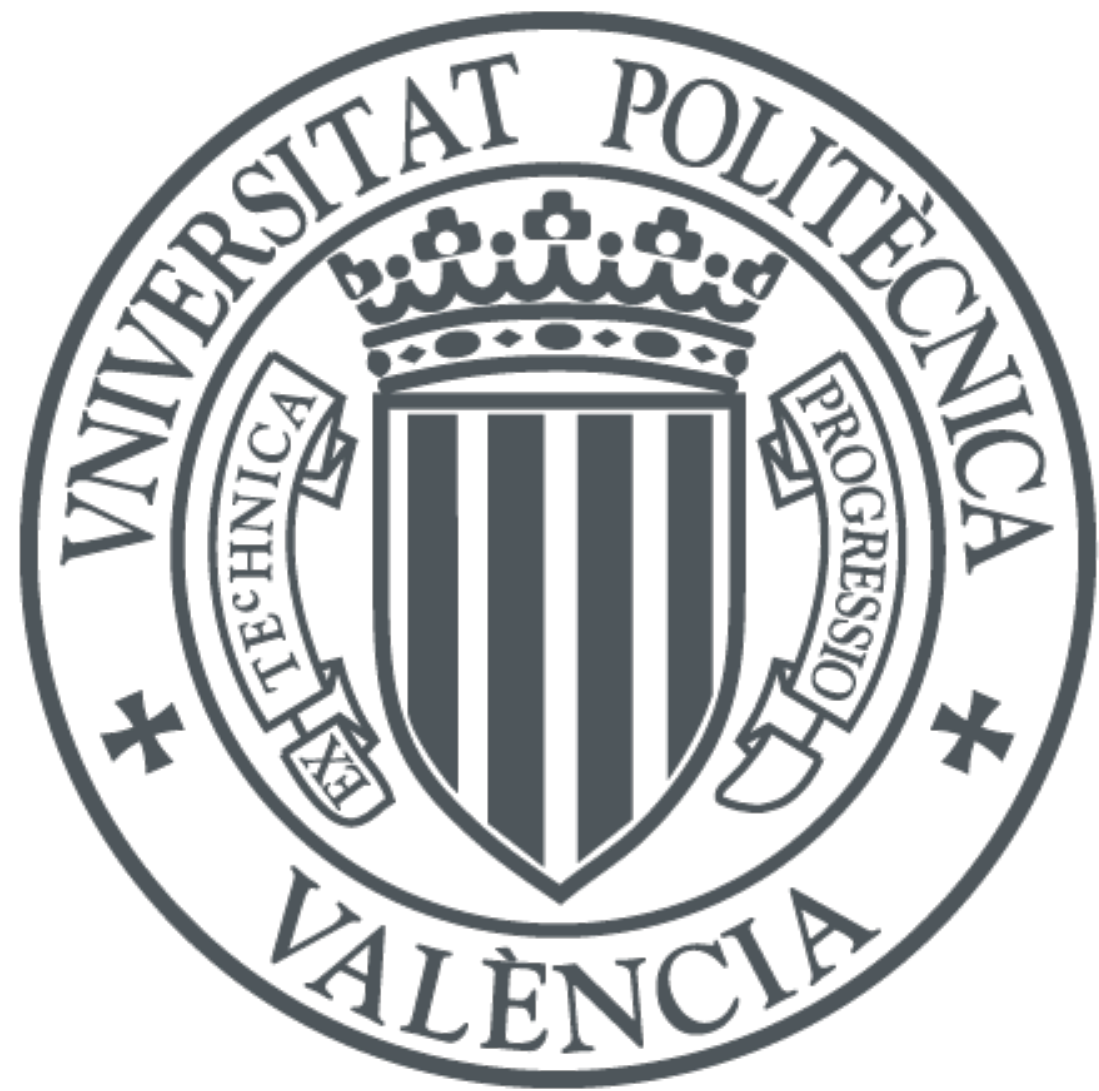

The final publication is available at

http://dx.doi.org/10.1109/TMTT.2014.2365477

Copyright Institute of Electrical and Electronics Engineers (IEEE)

Additional Information 
Document downloaded from:

http://hdl.handle.net/10251/56764

This paper must be cited as:

Sans, M.; Selga, J.; Rodríguez Pérez, AM.; Bonache, J.; Boria Esbert, VE.; Martín, F. (2014). Design of planar wideband bandpass filters from specifications using a two-step aggressive space mapping (ASM) optimization algorithm. IEEE Transactions on Microwave Theory and Techniques. 62(12):3341-3350. doi:10.1109/TMTT.2014.2365477

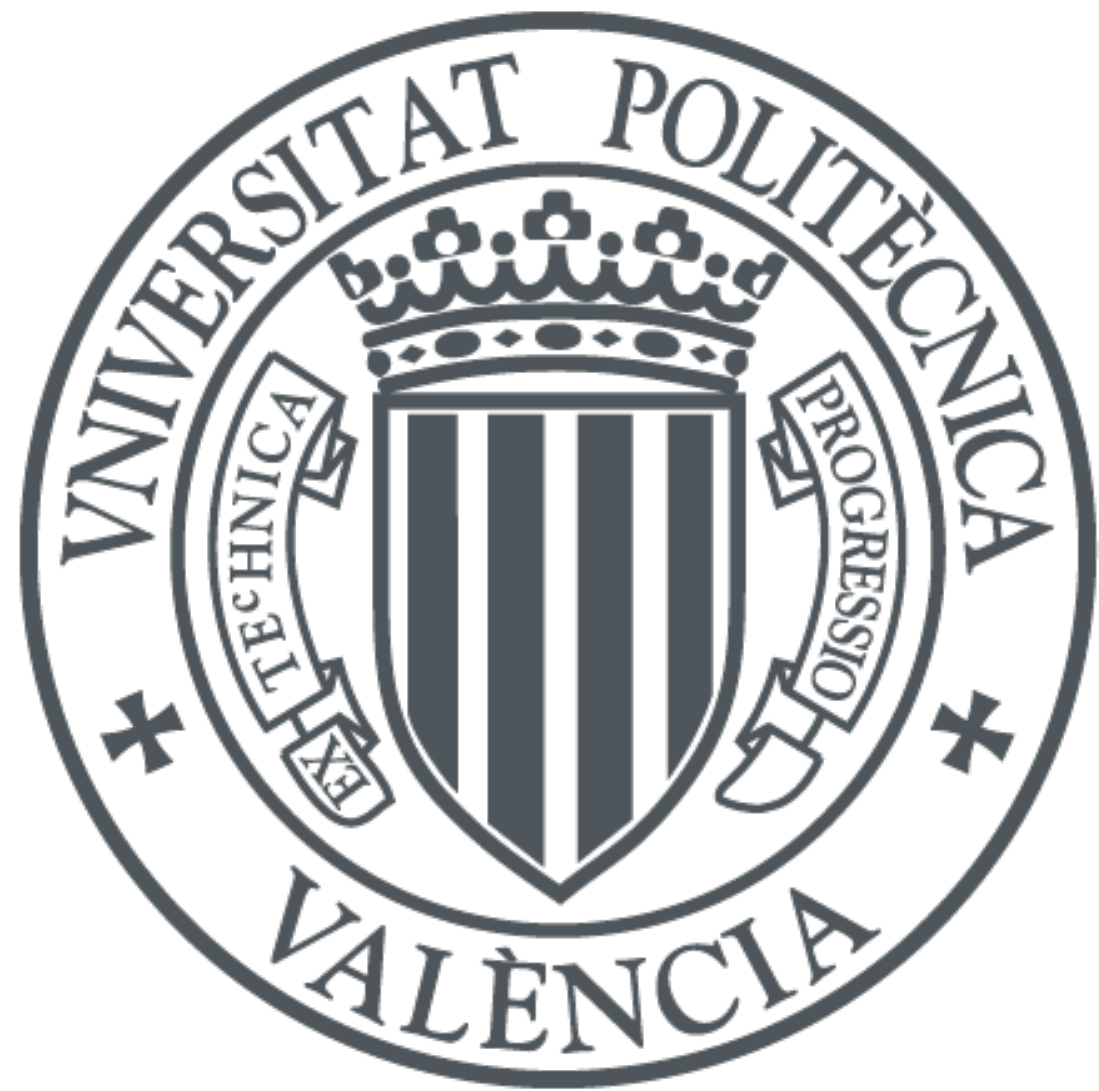

The final publication is available at

http://dx.doi.org/10.1109/TMTT.2014.2365477

Copyright Institute of Electrical and Electronics Engineers (IEEE)

Additional Information 


\title{
Design of Planar Wideband Bandpass Filters from Specifications Using a Two-Step Aggressive Space Mapping (ASM) Optimization Algorithm
}

\author{
Marc Sans, Jordi Selga, Member IEEE, Ana Rodríguez, Student Member IEEE, Jordi Bonache, Member \\ IEEE, Vicente E. Boria, Senior Member IEEE, and Ferran Martín, Fellow IEEE
}

\begin{abstract}
This paper is focused on the automated and unattended optimization of a type of planar wideband bandpass filters by means of aggressive space mapping (ASM). The considered filters are microstrip filters implemented through a combination of shunt connected stepped impedance resonators (SIRs) and grounded stubs coupled through admittance inverters. The most relevant and novel aspect of this paper is the fact that the filter layout is automatically generated from filter specifications, i.e., central frequency, fractional bandwidth, in-band ripple and order, without the need of any external aid to the design process. To achieve this layout generation, filter optimization has been divided into two independent ASM processes. The first one generates the filter schematic (circuit element values) providing the required specifications. This first iterative process is necessary since, due to the narrow band operation of the admittance inverters, the target specifications are achieved by compensating the effects of such narrow band operation. The purpose of the second ASM algorithm is to automatically generate the layout from the filter schematic resulting from the first ASM process. To validate the new two-step ASM optimization tool, two sets of filter specifications (inputs of the developed tool), are considered. The generated filter layouts exhibit frequency responses that satisfy the specifications, and are in excellent agreement with the responses of the schematics.
\end{abstract}

Index Terms- Bandpass filters, circuit optimization, microstrip technology, optimization, space mapping, stepped impedance resonators.

\section{INTRODUCTION}

The design of planar microwave components able to satisfy certain specifications has been a subject of interest for microwave engineers during years. Most commercial computer aided design (CAD) tools and electromagnetic solvers of planar circuits include optimizers. However, depending on the circuit complexity, it may not be so straightforward to find those topologies able to satisfy the design requirements. Namely, convergence to the optimum solution is not always guaranteed (for instance due to

This work has been supported by MINECO-Spain (projects TEC201017512 METATRANSFER, TEC2010-21520-C04-01 and TEC201347037-C5-1-R, CONSOLIDER EMET CSD2008-00066, TEC201340600-R and TEC2013-49221-EXP), Generalitat de Catalunya (project 2014SGR-157), and Institució Catalana de Recerca i Estudis Avançats (who has awarded Ferran Martín). This paper is an expanded paper from the IEEE MTT-S International Microwave Symposium, Tampa Bay, FL, USA, June 1-6, 2014

M. Sans, J. Selga, J. Bonache and F. Martín are with GEMMA/CIMITEC, Departament d'Enginyeria Electrònica, Universitat Autònoma de Barcelona, 08193 Bellaterra, Spain. E-mail: Ferran.Martin@uab.es.

A. Rodríguez and V.E. Boria are with Departamento de Comunicaciones-iTEAM, Universitat Politècnica de València, 46022 Valencia, Spain. E-mail: vboria@dcom.upv.es limitations related to local minima), unless the seeding layout is already very close to the one providing the target response.

Among the considered techniques for microwave circuit design and optimization, space mapping (SM), first proposed by Bandler et al. in 1994 [1], has revealed to be a powerful and efficient approach. Since that seminal work, several variants of SM have been proposed and applied to the design and optimization of many different microwave components, including not only planar circuits [2]-[4], but also waveguide-based components [5]-[7].

In this paper, the interest is focused on the so-called aggressive space mapping (ASM) [2], an approach that uses quasi-Newton type iteration to find the optimum solution of the considered problem. Particularly, the ASM optimization scheme will be adapted in order to find the layout of a class of bandpass filters (BPFs) that satisfies the design specifications. The considered filters are well suited to achieve wide fractional bandwidths, and can also be designed to exhibit high selectivity and wide stop bands by virtue of the controllable transmission zeros present above the pass band. Such filters consist of a combination of shunt connected stepped impedance resonators (SIRs) and grounded inductive stubs, coupled through admittance inverters [8]. Due to the narrow band operation of the admittance inverters (implemented by means of transmission line sections), ASM optimization will be first applied to the design of the optimum circuit schematic satisfying the specifications. Then, an additional (independent) ASM iterative process will be applied to the automatic generation of the filter layout. Hence, the proposed optimization tool will be able to automatically generate the filter layout from device specifications in a two-step ASM process. The optimum solution giving the target response is thus inferred following a completely unattended scheme.

The paper is organized as follows. The general formulation of ASM is briefly reviewed in Section II for completeness. Section III is focused on the first ASM algorithm, where the details to find the optimum filter schematic (the one that satisfies design requirements) are presented and discussed. This first ASM optimization algorithm constitutes the main contribution and originality of this paper. It provides the schematics of the considered filters satisfying the design specifications. Moreover, the proposed ASM algorithm can be easily modified in order to synthesize many other types of planar filters. Once the filter schematic is determined, a second ASM algorithm is used to derive the filter layout. The details and specificities of this algorithm, similar to the one reported in [9], are provided in section IV. For further clarification, Sections III 
and IV are supported by the design of an order-5 filter (the electromagnetic response of the filter is compared to measurements at the end of section IV). Nevertheless, to demonstrate the potential of the proposed optimization technique, Section $\mathrm{V}$ is focused on the design of an additional order-9 filter (also fabricated and measured). Finally, the main conclusions are highlighted in section VI.

\section{GENERAL FORMULATION OF AGGRESSIVE SPACE MAPPING (ASM)}

Space mapping (SM) is a technique extensively used for the design and optimization of microwave components. It uses two simulation spaces [1], [2], [10]: (i) the optimization space, $\mathbf{X}_{\mathbf{c}}$, where the variables are linked to a coarse model, which is simple and computationally efficient, although not accurate, and (ii) the validation space, $\mathbf{X}_{\mathbf{f}}$, where the variables are linked to a fine model, typically more complex and CPU intensive, but significantly more precise. In each space, a vector containing the different model parameters can be defined. Let us call such vectors $\mathbf{x}_{\mathbf{f}}$ and $\mathbf{x}_{\mathbf{c}}$ for the fine and coarse model spaces, respectively, and let us designate by $\mathbf{R}_{\mathbf{f}}\left(\mathbf{x}_{\mathbf{f}}\right)$ and $\mathbf{R}_{\mathbf{c}}\left(\mathbf{x}_{\mathbf{c}}\right)$ their corresponding responses. The key idea behind any SM optimization process is to generate an appropriate parameter transformation

$$
\mathbf{x}_{\mathbf{c}}=\mathbf{P}\left(\mathbf{x}_{\mathbf{f}}\right)
$$

mapping the fine model parameter space to the coarse model parameter space such that

$$
\left\|\mathbf{R}_{\mathbf{f}}\left(\mathbf{x}_{\mathbf{f}}\right)-\mathbf{R}_{\mathbf{c}}\left(\mathbf{x}_{\mathbf{c}}\right)\right\| \leq \eta
$$

in some predefined region, $\|\cdot\|$ being a certain suitable norm and $\eta$ a small positive number close to zero. If $\mathbf{P}$ is invertible, then the inverse transformation:

$$
\mathbf{x}_{\mathbf{f}}=\mathbf{P}^{-1}\left(\mathbf{x}_{\mathrm{c}}^{*}\right)
$$

is used to find the fine model solution, which is the image of the coarse model solution, $\mathbf{x}_{\mathbf{c}}{ }^{*}$, that gives the target response, $\mathbf{R}_{\mathbf{c}}\left(\mathbf{x}_{\mathbf{c}}{ }^{*}\right)$.

The determination of $\mathbf{P}$ according to the procedure reported in [1] follows an iterative process that is rather inefficient. The efficiency of the method was improved by introducing quasi-Newton type iteration [2], resulting in a faster convergence, and giving rise to the so-called aggressive space mapping (ASM) [2]. The goal in ASM is to minimize the following error function:

$$
\mathbf{f}\left(\mathbf{x}_{\mathbf{f}}\right)=\mathbf{P}\left(\mathbf{x}_{\mathbf{f}}\right)-\mathbf{x}_{\mathbf{c}}^{*}
$$

Let us assume that $\mathbf{x}_{\mathbf{f}}^{(j)}$ is the $j$-th approximation to the solution in the validation space, and $\mathbf{f}^{(j)}$ the error function corresponding to $\mathbf{f}\left(\mathbf{x}_{\mathbf{f}}^{(j)}\right)$. The next vector of the iterative process $\mathbf{x}_{\mathbf{f}}^{(j+1)}$ is obtained by a quasi-Newton iteration according to

$$
\mathbf{x}_{\mathbf{f}}^{(j+1)}=\mathbf{x}_{\mathbf{f}}^{(j)}+\mathbf{h}^{(j)}
$$

where $\mathbf{h}^{(j)}$ is given by:

$$
\mathbf{h}^{(j)}=-\left(\mathbf{B}^{(j)}\right)^{-1} \mathbf{f}^{(j)}
$$

and $\mathbf{B}^{(j)}$ is an approach to the Jacobian matrix, which is updated according to the Broyden formula [2]:

$$
\mathbf{B}^{(j+1)}=\mathbf{B}^{(j)}+\frac{\mathbf{f}^{(j+1)} \mathbf{h}^{(j) T}}{\mathbf{h}^{(j) T} \mathbf{h}^{(j)}}
$$

In (7), $\mathbf{f}^{(j+1)}$ is obtained by evaluating (4), using a certain parameter extraction method providing the coarse model parameters from the fine model parameters, and the superindex $T$ stands for transpose.
The implementation of the ASM algorithm is well reported in [2]. In the next two sections, the details of the two ASM algorithms involved in the automated design of the filters considered in this work are discussed in detail.

\section{FIRST ASM ALGORITHM: DETERMINATION OF THE OPTIMUM FILTER SCHEMATIC}

In this section we first present the considered wideband bandpass filters, including their schematic and topology, and then we describe the proposed ASM algorithm for the determination of the filter schematic that satisfies the design specifications.

\section{A. Schematic and Topology of the Considered Wideband Bandpass Filters}

The filters to be synthesized in this work are based on shunt resonators coupled through admittance inverters (see Fig. 1), according to the well-known network of a bandpass filter reported in many textbooks [11]. In order to achieve wide fractional bandwidth, high selectivity at the upper transition band and spurious free responses over broad frequency bands, the shunt resonators are implemented through a combination of stepped impedance resonators (SIRs) and grounded stubs. The SIRs provide transmission zeros (at frequencies designated as $f_{z}$ ) above the central filter frequency, $f_{0}$, which are useful for spurious suppression and for achieving a pronounced fall-off above the upper band edge. Moreover, with the parallel combination of SIRs and inductive stubs, the susceptance slopes at the filter central frequency can be made small, as required to achieve broad fractional bandwidths [8].

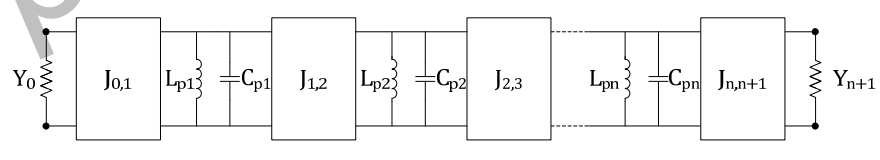

Fig. 1. Generalized bandpass filter network based on shunt resonators coupled through admittance inverters.

The typical topology and circuit schematic of the filter are depicted in Fig. 2, where the SIRs are described by the resonators $L_{r i}-C_{r i}$, and $L_{p i}$ accounts for the grounded stubs ( $i$ denotes the filter stage). The admittance inverters of the network of Fig. 1 are usually implemented by means of quarter wavelength (at $f_{0}$ ) transmission line sections. If the filter is narrowband, the target response (i.e., the one provided by the schematic considering ideal admittance inverters) can be achieved to a good approximation by means of quarter wavelength transmission lines. However, in wideband filters the narrowband functionality of these lines as admittance inverters degrades (decreases) the fractional bandwidth, and modifies the central frequency and the in-band return loss level. To solve this problem, it is necessary to tailor the parameters of the circuit schematic of Fig. 2(b), that is, the reactive parameters $\left(L_{r i}, C_{r i}\right.$ and $\left.L_{p i}\right)$ and the electrical lengths of the transmission line sections. In the next subsection, we will present an ASM-based algorithm that automatically re-calculates these parameters in order to satisfy the filter specifications. The generated schematic after this iterative ASM process will be designated as the optimum filter schematic. 


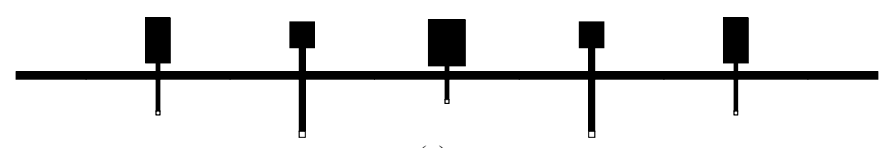

(a)

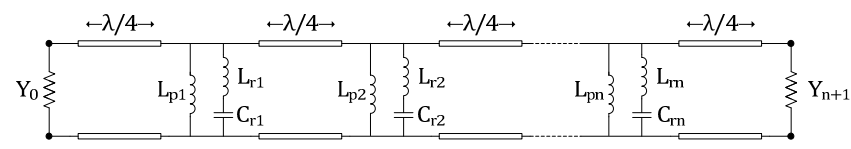

(b)

Fig. 2. Typical topology (a) and circuit schematic (b) of the considered wideband bandpass filters. The topology corresponds to an order- 5 filter.

\section{B. Implementation of the ASM Algorithm}

Let us consider that the filter order, $n$, is set to a certain value that suffices to achieve the required filter selectivity. The filter specifications are the central frequency, $f_{0}$, the fractional bandwidth, $F B W$, and the in-band ripple level $L_{A r}$ (or minimum return loss level). The transmission zero frequencies provided by the SIRs are all set to $f_{z}=2 f_{0}$, since this has been demonstrated to be a convenient approach to achieve spurious suppression, and to improve filter selectivity above the upper band edge [8],[9]. From the well-known impedance and frequency transformations from the low pass filter prototype [11], and assuming a Chebyshev response, the reactive elements of the shunt resonators of the network of Fig. $2\left(L_{r i}, C_{r i}\right.$, and $\left.L_{p i}\right)$ can be easily inferred (the admittance of the inverters is set to $J=$ $0.02 \mathrm{~S}$ ). The three conditions to unequivocally determine $L_{r}$, $C_{r}$, and $L_{p}$ are: (i) the filter central frequency, given by

$$
f_{0}=\frac{1}{2 \pi \sqrt{\left(L_{r i}+L_{p i}\right) C_{r i}}}
$$

(ii) the transmission zero frequency

$$
f_{z}=\frac{1}{2 \pi \sqrt{L_{r i} C_{r i}}}
$$

and (iii) the susceptance slope at $f_{0}$ (dependent on the filter stage):

$$
b_{i}=2 \pi f_{0} \frac{C_{r i}\left(L_{r i}+L_{p i}\right)^{2}}{L_{p i}^{2}}
$$

Considering that the target is an order-5 $(n=5)$ Chebyshev response with $f_{0}=2.4 \mathrm{GHz}, F B W=40 \%$ (corresponding to a $43.96 \%-3-\mathrm{dB}$ fractional bandwidth) and $L_{A r}=0.2 \mathrm{~dB}$, the element values of the shunt resonators are found to be those indicated in Table I. It is important to mention that for Chebyshev bandpass filters the fractional bandwidth is given by the ripple level and is hence smaller than the $-3-\mathrm{dB}$ fractional bandwidth. However, in this paper we will deal with the $-3-\mathrm{dB}$ fractional bandwidth since the ripple level is not constant in the optimization process (to be described). From now on, this $-3-\mathrm{dB}$ fractional bandwidth is designated as $F B W$, rather than $F B W_{-3 d B}$ (as usual), for simplicity, and to avoid an excess of subscripts in the formulation.

The quasi-Chebyshev filter response (i.e. the one inferred from the schematic of Fig. 2(b), but with ideal admittance inverters), depicted in Fig. 3, is similar to the ideal (target) Chebyshev response in the pass band region, and it progressively deviates from it as frequency approaches $f_{z}$, as expected. The discrepancies are due to the fact that the shunt resonator is actually a combination of a grounded series resonator (providing the transmission zero) and a grounded inductor. The quasi-Chebyshev response satisfies the specifications to a rough approximation. Hence the target is considered to be the ideal Chebyshev response, except for the transmission zero frequency. In Fig. 3, the responses have been obtained by implementing a specific code, implemented in $M A T L A B^{\circledR}$ [12], that calculates the transfer $(A B C D)$ matrix of the filter from the individual matrices of the different filter sections.

TABLE I

ELEMENT VALUES OF THE SHUNT RESONATORS

\begin{tabular}{|c|c|c|c|}
\hline Stage & $\boldsymbol{L}_{\boldsymbol{p}}(\mathrm{nH})$ & $\boldsymbol{L}_{\boldsymbol{r}}(\mathrm{nH})$ & $\boldsymbol{C}_{\boldsymbol{r}}(\mathrm{pF})$ \\
\hline 1,5 & 1.3202 & 0.4401 & 2.4983 \\
\hline 2,4 & 1.3226 & 0.4409 & 2.4937 \\
\hline 3 & 0.8164 & 0.2721 & 4.0400 \\
\hline
\end{tabular}

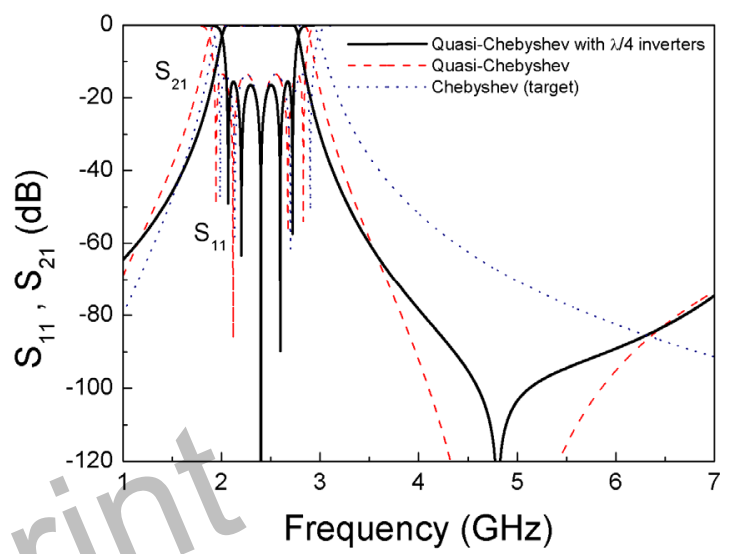

Fig. 3. Quasi-Chebyshev response of the filter that results by using the element values of Table I and ideal admittance inverters (red dashed line), compared to the filter response that results by replacing the ideal inverters with quarter wavelength transmission lines (black solid line), and with the ideal Chebyshev (target) response.

If we now replace the ideal admittance inverters with quarter wavelength transmission lines, the response is further modified, as Fig. 3 illustrates. Thus, our aim is to find the filter schematic [Fig. 2(b)] able to satisfy the specifications. To this end, a new ASM concept that carries out the optimization at the schematic level is proposed in this work.

The key point in the development of this novel iterative ASM algorithm, is to assume that there is a set of filter specifications, different from the target, that leads to a filter schematic (inferred by substituting the ideal admittance inverters with quarter wavelength transmission lines), whose response satisfies the target specifications. Let us now try to define the optimization (coarse model) space and the validation (fine model) space in the proposed ASM iterative scheme. The first one is constituted by the set of specifications, $f_{0}, F B W, L_{A r}$, being its response the ideal Chebyshev response -target response- depicted in Fig. 3. The validation space is constituted by the same variables, but their response is inferred from the schematic of Fig. 2(b), with element values calculated as specified above, and quarter wavelength transmission lines at $f_{0}$, where $f_{0}$ is the considered value of this element in the validation space (not necessarily the target filter central frequency). The variables of each space are differentiated by a subscript. Thus, the corresponding vectors in the coarse and fine models are written as $\mathbf{x}_{\mathbf{c}}=\left[f_{0 \mathrm{c}}, F B W_{\mathrm{c}}, L_{A r \mathrm{c}}\right]$ and $\mathbf{x}_{\mathbf{f}}=\left[f_{0 \mathrm{f}}, F B W_{\mathrm{f}}, L_{A r \mathrm{f}}\right]$, respectively. The coarse model solution (target 
specifications) is expressed as $\mathbf{x}_{\mathbf{c}}{ }^{*}=\left[f_{0 \mathrm{c}}{ }^{*}, F B W_{\mathrm{c}}{ }^{*}, L_{A r \mathrm{c}}{ }^{*}\right]$ Notice that the transmission zero frequency, necessary to unequivocally determine the element values of the shunt resonators, is set to $f_{z}=2 f_{0}$, as indicated before. Hence $f_{z}$ is not a variable in the optimization process.

Following the standard procedure in ASM, the first step before starting the iterative process is to make an estimation of the first vector in the validation space, $\mathbf{x}_{\mathbf{f}}{ }^{(1)}$. Since the variables in both spaces are the same, the most canonical (and simplest) procedure is to consider $\mathbf{x}_{\mathbf{f}}{ }^{(1)}=\mathbf{x}_{\mathbf{c}}{ }^{*}$. From $\mathbf{x}_{\mathbf{f}}{ }^{(1)}$, the response of the fine model space is obtained (using the schematic with quarter wavelength transmission lines), and from it we directly extract the parameters of the coarse model by direct inspection of that response, i.e., $\mathbf{x}_{\mathbf{c}}{ }^{(1)}=$ $\mathbf{P}\left(\mathbf{x}_{\mathbf{f}}{ }^{(1)}\right)$. Applying (4), we can thus obtain the first error function. To iterate the process [obtaining $\mathbf{x}_{\mathbf{f}}^{(2)}$ from (5), using (6)], we need to initiate the Jacobian matrix. To this end, we have slightly perturbed the parameters of the fine model, $f_{0 \mathrm{f}}, F B W_{\mathrm{f}}, L_{A r \mathrm{f}}$, and we have inferred the effects of such perturbations on the coarse model parameters, $f_{0 \mathrm{c}}$, $F B W_{\mathrm{c}}, L_{A r \mathrm{c}}$. Thus, the first Jacobian matrix is given by:

$$
\mathbf{B}=\left(\begin{array}{ccc}
\frac{\delta f_{0 \mathrm{c}}}{\delta f_{0 \mathrm{f}}} & \frac{\delta f_{0 \mathrm{c}}}{\delta F B W_{\mathrm{f}}} & \frac{\delta f_{0 \mathrm{c}}}{\delta L_{A r \mathrm{f}}} \\
\frac{\delta F B W_{\mathrm{c}}}{\delta f_{0 \mathrm{f}}} & \frac{\delta F B W_{\mathrm{c}}}{\delta F B W_{\mathrm{f}}} & \frac{\delta F B W_{\mathrm{c}}}{\delta L_{A r \mathrm{f}}} \\
\frac{\delta L_{A r \mathrm{c}}}{\delta f_{0 \mathrm{f}}} & \frac{\delta L_{A r \mathrm{c}}}{\delta F B W_{\mathrm{f}}} & \frac{\delta L_{A r \mathrm{c}}}{\delta L_{A r \mathrm{f}}}
\end{array}\right)
$$

Once the first Jacobian matrix is obtained, the process can be iterated until convergence is obtained. At each iteration, the elements of the coarse space vector, $\mathbf{x}_{\mathbf{c}}{ }^{(j)}$, are compared to the target (filter specifications), $\mathbf{x}_{\mathbf{c}}{ }^{*}$, and the error function is obtained according to:

$$
\left\|f_{\text {norm }}\right\|=\sqrt{\left(1-\frac{f_{0 \mathrm{c}}}{f_{0 \mathrm{c}}^{*}}\right)^{2}+\left(1-\frac{F B W_{\mathrm{c}}}{F B W_{\mathrm{c}}^{*}}\right)^{2}+\left(1-\frac{L_{A r \mathrm{c}}}{L_{A r \mathrm{c}}^{*}}\right)^{2}}
$$

The scheme of the proposed ASM algorithm is depicted in Fig. 4. The core program has been written in MATLAB ${ }^{\circledR}$ [12], and the fine model responses have been obtained, also using MATLAB ${ }^{\circledR}$, by first calculating the $A B C D$ matrix and transforming it to the scattering matrix.
Applying the developed ASM algorithm to the considered example $\left(\mathbf{x}_{\mathrm{c}}{ }^{*}=\left[f_{0 \mathrm{c}}{ }^{*}, F B W_{\mathrm{c}}{ }^{*}, L_{A r c}{ }^{*}\right]=[2.4 \mathrm{GHz}, 43.96 \%, 0.2\right.$ $\mathrm{dB}]$ ), we have found that the error function rapidly decreases, reducing the error to around $11 \%$ after iteration $N=2$, and smaller than $1.2 \%$ after $N=13$ iterations. The evolution of the error function is depicted in Fig. 5. The fine model parameters for $N=2$ are $\mathbf{x}_{\mathbf{f}}^{(2)}=\left[f_{0 \mathrm{f}}^{(2)}, F B W_{\mathrm{f}}^{(2)}, L_{A r f}{ }^{(2)}\right]$ $=[2.4690 \mathrm{GHz}, 65.37 \%, 0.3902 \mathrm{~dB}]$ and the coarse model parameters are $\mathbf{x}_{\mathbf{c}}{ }^{(2)}=\left[f_{0 \mathrm{c}}{ }^{(2)}, F B W_{\mathrm{c}}^{(2)}, L_{A r \mathrm{c}}{ }^{(2)}\right]=[2.3998 \mathrm{GHz}$, $43.75 \%, 0.1780 \mathrm{~dB}]$, whereas for the last iteration $(N=13)$ the fine model parameters are $\mathbf{x}_{\mathbf{f}}{ }^{(13)}=\left[f_{\text {of }}{ }^{(13)}, F B W_{\mathrm{f}}^{(13)}\right.$, $\left.L_{A r f}{ }^{(13)}\right]=[2.4690 \mathrm{GHz}, 65.53 \%, 0.4413 \mathrm{~dB}]$ and the coarse model parameters are $\mathbf{x}_{\mathbf{c}}{ }^{(13)}=\left[f_{0 \mathrm{c}}{ }^{(13)}, F B W_{\mathrm{c}}{ }^{(13)}, L_{A r c}{ }^{(13)}\right]=$ $[2.3999 \mathrm{GHz}, 43.75 \%, 0.1978 \mathrm{~dB}]$. Thus in both iterations, $\mathbf{x}_{\mathbf{f}}$ is appreciably different than $\mathbf{x}_{\mathbf{c}}{ }^{*}$. The optimum filter schematic is the one given by the last fine model response (which provides an error below a predefined value). The elements of the shunt resonators for this optimum filter schematic are indicated in Table II, whereas the 50- $\Omega$ line sections are quarter wavelength transmission lines at $f_{0 \mathrm{f}}{ }^{(13)} \neq$ $f_{0}^{*}=2.4 \mathrm{GHz}$. The response of the optimum schematic is compared to the target response in Fig. 6. The agreement in terms of central frequency, bandwidth and in-band ripple is very good, as expected on account of the small error function that has been obtained after 13 iterations. Note, however, that the position of the reflection zero frequencies are different in both responses. The reason is that we have not considered these frequency positions as goals in the optimization process. Unavoidably, it is not possible to perfectly match the Chebyshev (target) response by replacing the ideal admittance inverters with transmission line sections and the LC shunt resonators of Fig. 1 with the resonators of the schematic of Fig. 2(b). Nevertheless the synthesized circuit fulfills the target specifications, and hence it is the optimum filter schematic used as the starting point in the ASM algorithm developed to obtain the filter layout, to be described in the next section.

TABLE II

ELEMENT VALUES OF THE SHUNT RESONATORS FOR THE OPTIMUM FILTER SCHEMATIC

\begin{tabular}{|c|c|c|c|}
\hline Stage & $\boldsymbol{L}_{\boldsymbol{p}}(\mathrm{nH})$ & $\boldsymbol{L}_{\boldsymbol{r}}(\mathrm{nH})$ & $\boldsymbol{C}_{\boldsymbol{r}}(\mathrm{pF})$ \\
\hline 1,5 & 1.6090 & 0.5363 & 1.9368 \\
\hline 2,4 & 2.1196 & 0.7065 & 1.4703 \\
\hline 3 & 1.0685 & 0.3562 & 2.9168 \\
\hline
\end{tabular}



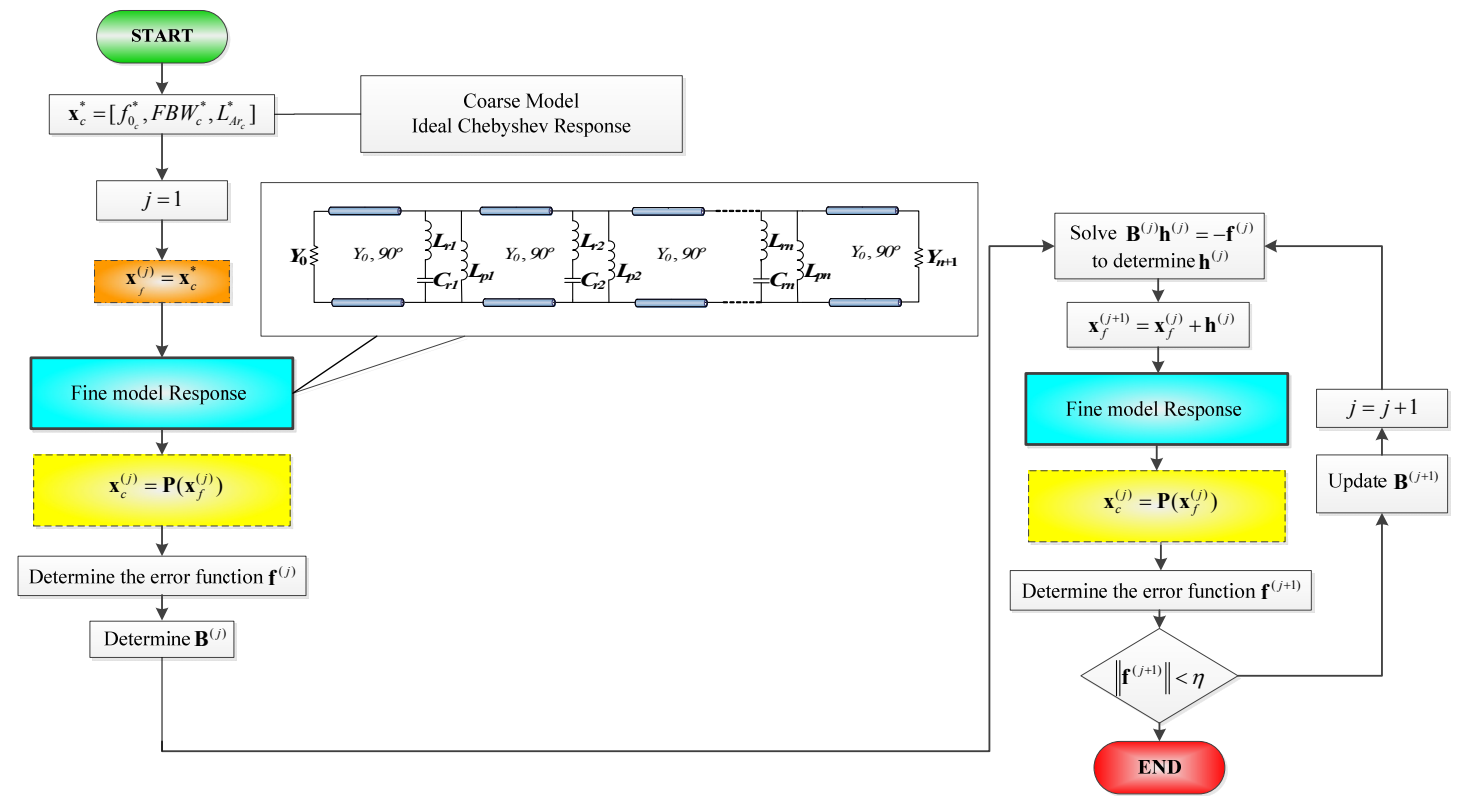

Fig. 4. Flow diagram of the first ASM algorithm.

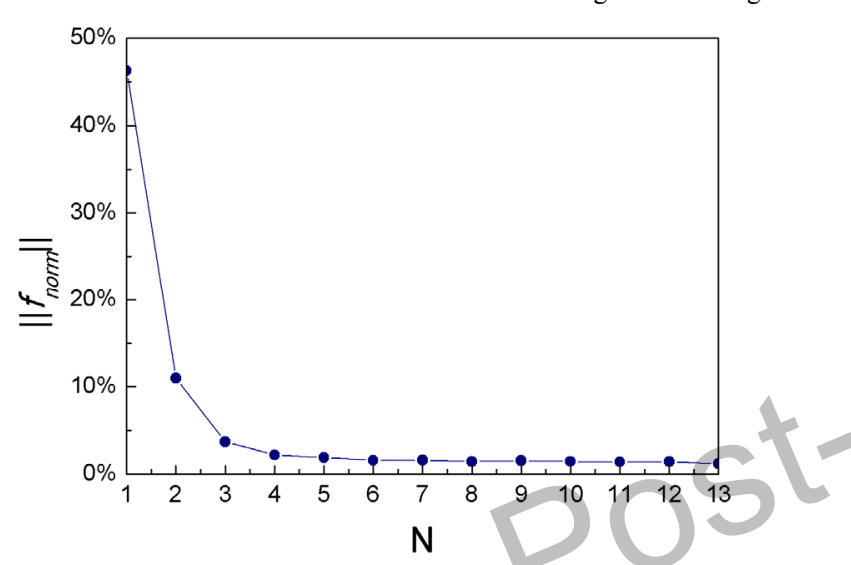

Fig. 5. Evolution of the error function of the first ASM algorithm for the considered example.

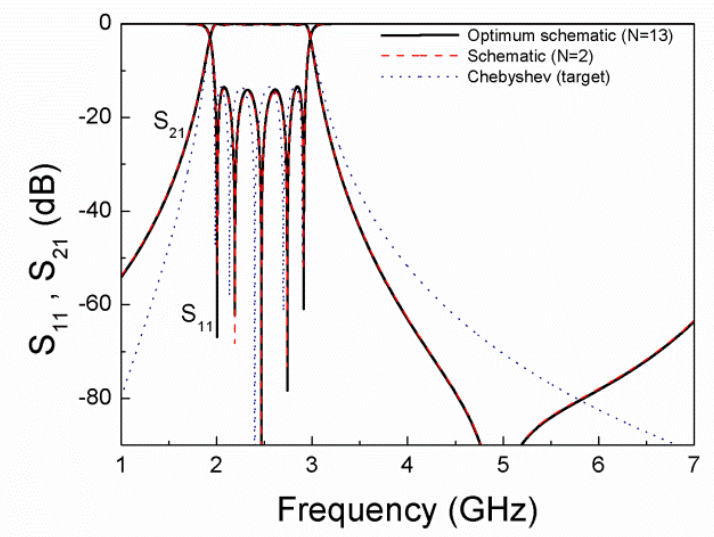

Fig. 6. Response of the optimum filter schematic, derived from the ASM algorithm, compared with the Chebyshev target response. The response at the second iteration $(N=2)$, also included, is very close to the final solution $(N=13)$.

To end this section, we would like to highlight that the previous procedure to find the optimum filter schematic, based on an iterative ASM algorithm, represents clear progress as compared to the method reported in [9], and it is the main relevant contribution of the present paper. In [9], we simply applied an iterative process to find the optimum schematic, involving only one variable: the fractional bandwidth. We iteratively readjusted the nominal fractional bandwidth until the resulting response (at the schematic level) converged to the required bandwidth. However, by means of such procedure we cannot guarantee that the inband ripple is within the required level. Conversely, through the ASM-based method proposed in this paper it has been demonstrated that the filter specifications (central frequency, fractional bandwidth and in-band ripple) can be fully satisfied by the automatically generated filter schematic. The reason is that the new developed ASM involves the previous set of specifications as variables in the optimization process.

\section{SECOND ASM ALGORITHM: AUTOMATED LAYOUT GENERATION}

Once the optimum filter schematic has been found, the next step is to determine the layout of the filter. To this end, a second ASM algorithm, firstly reported in [9], is considered. We have to synthesize each filter cell from the element values of the shunt resonator and the characteristic impedance of the cascaded $\lambda / 8$ (at $f_{0 \mathrm{f}}{ }^{(13)}$ ) transmission lines independently. This second ASM process involves three stages: (i) determination of the resonator layout, (ii) determination of the line width, and (iii) optimization of the line length. Let us now discuss in detail the implementation of these three specific ASM algorithms.

\section{A. Optimization of the Resonators}

In order to obtain the layout of the filter resonators, composed by the SIRs parallel connected to the grounded stubs, a specific ASM iterative process has been designed. The variables in the optimization space are the resonator elements, i.e., $\mathbf{x}_{\mathbf{c}}=\left[L_{p}, L_{r}, C_{r}\right]$, and the coarse model response is obtained through circuit simulation. The validation space is constituted by a set of variables defining the resonator layout. In order to deal with the same number of variables in both spaces, the widths of the grounded stubs, $W_{L p}$, as well as the widths of the low- and highimpedance transmission line sections of the SIRs, $W_{C r}$ and $W_{L r}$, respectively, are set to fixed values. Specifically, we have considered the values of $W_{C r}$ and $W_{L r}=W_{L p}$ indicated in tables III and V. There is some flexibility to choose these widths, but it is convenient to consider wide capacitive and 
narrow inductive sections to reduce the length of the SIRs and inductive stubs. We have opted in this work to consider moderate widths of the capacitive sections to avoid transversal resonances in the frequency region of interest (i.e., up to frequencies above $f_{z}$ ). The widths of the inductive sections have been set to values above the tolerance limits (representing a good balance between $\mathrm{SIR} /$ stub dimensions and fabrication accuracy). Thus, the variables in the validation space are the remaining dimensions of the shunt resonators, that is, the length of the grounded stub, $l_{L p}$, and the lengths $l_{L r}$ and $l_{C r}$ of the highand low-impedance transmission line sections, respectively, of the SIR (i.e., $\mathbf{x}_{\mathbf{f}}=\left[l_{L p}, l_{L r}, l_{C r}\right]$ ). The fine model response is obtained through electromagnetic simulation of the layout, inferred from the fine model variables plus the fixed dimensions (specified above) and substrate parameters. In this work, the Agilent Momentum commercial software has been used to obtain the electromagnetic response of the structures, and the considered substrate parameters are those of the Rogers RO3010 with thickness $h=635 \mu \mathrm{m}$ and dielectric constant $\varepsilon_{r}=10.2$.

Following the general scheme of the ASM optimization described in section II, to initiate the algorithm it is necessary to obtain an initial layout for the SIR and shunt stub. This is obtained from the well-known (and simple) approximate formulas providing the inductance and capacitance of a narrow and wide, respectively, electrically small transmission line section [14]. Isolating the lengths, we obtain:

$$
\begin{aligned}
& l_{L r}=\frac{L_{r} v_{p h}}{Z_{h}} \\
& l_{C r}=C_{r} v_{p l} Z_{l} \\
& l_{L p}=\frac{L_{p} v_{p h}}{Z_{h}}
\end{aligned}
$$

where $v_{p h}$ and $v_{p l}$ are the phase velocities of the high- and low-impedance transmission lines sections, respectively, and $Z_{h}$ and $Z_{l}$ the corresponding characteristic impedances. Once the initial layout (i.e., $\mathbf{x}_{\mathbf{f}}^{(1)}$ ) is determined, the circuit elements can be extracted from the electromagnetic response using (8)-(10). This provides $\mathbf{x}_{\mathbf{c}}^{(1)}=\mathbf{P}\left(\mathbf{x}_{\mathbf{f}}^{(1)}\right)$, and using (4), the first error function can be inferred. To iterate the process using (5), with $\mathbf{h}^{(1)}$ derived from (6), a first approximation of the Jacobian matrix is needed. Following a similar approach to the one explained in Section III.B, we have slightly perturbed the lengths $l_{L p}, l_{L r}, l_{C r}$, and we have obtained the values of $L_{p}, L_{r}$ and $C_{r}$ resulting after each perturbation from parameter extraction. The first Jacobian matrix can thus be expressed as:

$$
\mathbf{B}=\left(\begin{array}{lll}
\frac{\delta L_{r}}{\delta l_{L r}} & \frac{\delta L_{r}}{\delta l_{C r}} & \frac{\delta L_{r}}{\delta l_{L p}} \\
\frac{\delta C_{r}}{\delta l_{L r}} & \frac{\delta C_{r}}{\delta l_{C r}} & \frac{\delta C_{r}}{\delta l_{L p}} \\
\frac{\delta L_{p}}{\delta l_{L r}} & \frac{\delta L_{p}}{\delta l_{C r}} & \frac{\delta L_{p}}{\delta l_{L p}}
\end{array}\right)
$$

By applying this procedure to the resonator elements of each filter stage, the corresponding layouts of the SIRs and grounded stubs are determined.

\section{B. Determination of the Line Width}

The optimization of the quarter wavelength (at $f_{0 \mathrm{f}}{ }^{(13)}$ ) transmission lines is much simpler, since it involves only the line width, $W$, that must be determined from the target characteristic impedance $\left(Z_{0}=50 \Omega\right)$. The line length is not subjected to optimization at this stage, since the presence of the resonator is expected to cause some extra phase shift. Therefore the line length is optimized in the next stage, where the required phase shift is forced in the whole structure, including the pair of lines and the resonator.

The initial line width is estimated from the formulas provided in several microwave textbooks (for instance [15]). Once the initial width is estimated, the specific ASM algorithm developed to determine the line width is applied. In such one-variable ASM scheme, the initial Jacobian matrix (actually just composed of one element) is inferred by perturbing the line width and obtaining the characteristic impedance through electromagnetic simulation (i.e., the fine model variable is the line width, $W$, whereas the variable of the coarse model is the characteristic impedance).

\section{Optimization of the Line Length (Filter Cell Optimization)}

As mentioned before, the length of the lines cascaded to the resonant elements is optimized by considering the whole filter cell. Let us define $l_{\text {cell }}$ as the length of the cell excluding the width of the grounded stubs, $W_{L p}$, (roughly corresponding to $\lambda / 4$ at $f_{0 \mathrm{f}}^{(13)}$ ). To determine $l_{\text {cell }}$, a single parameter ASM optimization is applied to the filter cell (filter cell optimization), where the initial value of $l_{\text {cell }}$ is inferred from the well-known formula [14] that gives the line length as a function of the required phase $\left(90^{\circ}\right)$ and frequency $\left(f_{0 \mathrm{f}}^{(13)}\right)$. At this stage, the ASM optimization consists of varying the length of the lines cascaded to the resonator until the required phase at $f_{0 \mathrm{f}}^{(13)}$ (i.e., $90^{\circ}$ ) per filter cell is achieved (the other geometrical parameters of the cell are kept unaltered). The phase is directly inferred from the frequency response of the cell obtained from electromagnetic simulation at each iteration step.

Once each filter cell has been synthesized, the cells are simply cascaded to generate the final filter layout (coupling between adjacent resonators is not taken into account since the results reveal that this effect is not significant). The scheme of the complete ASM process able to automatically provide the layout from the optimum filter schematic, and consisting of three independent quasi-Newton iterative algorithms, is depicted in Fig. 7. Using the element values of Table II, corresponding to the optimum filter schematic of the example reported in section III, where the lines present between adjacent resonators exhibit a characteristic impedance of $Z_{o}=50 \Omega$ and an electrical length of $90^{\circ}$ at $f_{\text {of }}^{(13)}=2.4690 \mathrm{GHz}$, we have applied the developed ASM algorithm to automatically generate the filter layout (which is actually the one depicted in Fig. 2). The dimensions are summarized in Table III, except the line width, which does not depend on the filter stage, i.e., $W=0.6055 \mathrm{~mm}$. Notice that the cell length slightly varies from cell to cell. This variation is due to the phase effects produced by the different resonators, and justifies the need to optimize the length of the lines by considering the complete filter cell (as described in the preceding paragraph). 


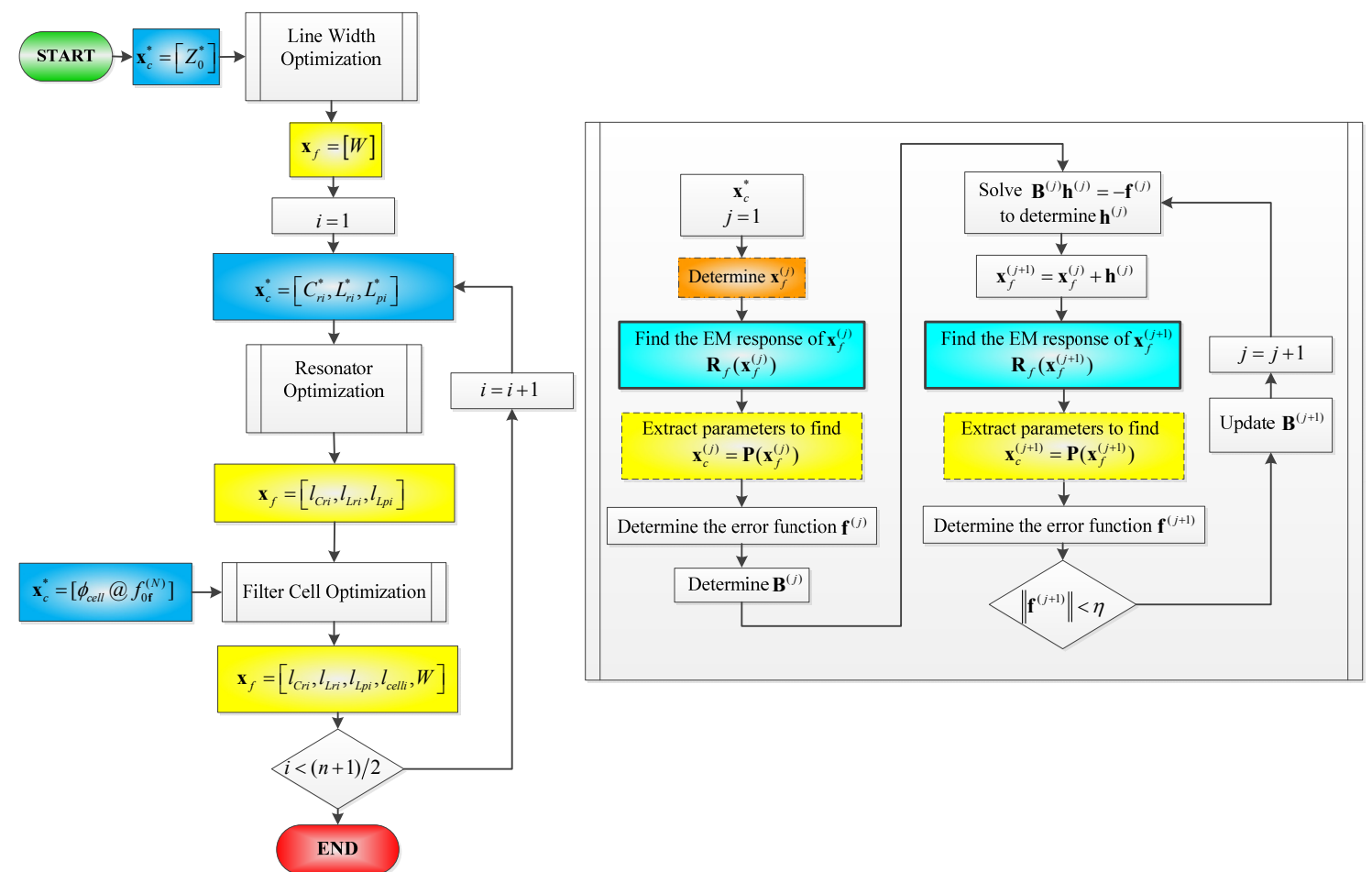

Fig. 7. Flow diagram of the second ASM algorithm. The sub-process depicted at the right of the figure represents a typical ASM algorithm used in each optimization process (particularly the indicated one is for the resonator optimization). Notice that the loop must be executed ( $n+1) / 2$ times, $n$ being the filter order (which is assumed to be odd), since for odd Chebyshev response the cells $i$ and $n+1-i$ are identical. However, this does not affect line width optimization since line width is identical for all filter stages.

TABLE III

GEOMETRY PARAMETERS OF THE SYNTHESIZED ORDER-5 FILTER

\begin{tabular}{|c|c|c|c|c|c|c|}
\hline Stage & $\begin{array}{c}\boldsymbol{l}_{\boldsymbol{L r}} \\
(\mathrm{mm})\end{array}$ & $\begin{array}{c}\boldsymbol{l}_{\boldsymbol{C r}} \\
(\mathrm{mm})\end{array}$ & $\begin{array}{c}\boldsymbol{l}_{\boldsymbol{L p}} \\
(\mathrm{mm})\end{array}$ & $\begin{array}{c}\boldsymbol{I}_{\text {cell }} \\
(\mathrm{mm})\end{array}$ & $\begin{array}{c}\boldsymbol{W}_{\boldsymbol{C r}} \\
(\mathrm{mm})\end{array}$ & $\begin{array}{c}\boldsymbol{W}_{\boldsymbol{L p}} \\
(\mathrm{mm})\end{array}$ \\
\hline 1,5 & 0.7062 & 3.6710 & 2.5955 & 11.4003 & 2 & 0.3 \\
\hline 2,4 & 1.9438 & 2.1037 & 4.2334 & 11.2583 & 2 & 0.5 \\
\hline 3 & 0.4725 & 3.7461 & 1.6629 & 11.4786 & 3 & 0.3 \\
\hline
\end{tabular}

The electromagnetic simulation (excluding losses) of the synthesized filter is compared to the response of the optimum filter schematic and to the target (ideal Chebyshev) response in Fig. 8. The agreement between the lossless electromagnetic simulation and the response of the optimum filter schematic (where losses are excluded) is very good, pointing out the validity of the second ASM optimization method. The filter has been fabricated by means of an LPKF H100 drilling machine (see Fig. 9(a)), and the measured frequency response (Fig. 9(b)) has been obtained by means of an Agilent N5221A vector network analyzer. The measured response is in reasonable agreement with the lossy electromagnetic simulation, and reveals that filter specifications are satisfied to a good approximation. Slight discrepancies between the measured response and the lossy electromagnetic simulation can be mainly attributed to inaccuracies in the dielectric constant provided by the substrate supplier, although they can be also influenced by fabrication related tolerances, substrate anisotropy and foil roughness. Nevertheless, these effects are not considered in the optimization process, because the aim was to automatically obtain the filter layout providing a lossless electromagnetic simulation able to satisfy the specifications. Hence, the results of Fig. 8 reveal that this objective has been fully achieved. Note also that the effects of placing the filter in a shielding box are not taken into account in this work (filter response might be affected by box resonances if they are present in the region of interest).

It is also worth highlighting that layout generation with the proposed two-step ASM procedure (design and optimization of the filter schematic and of the filter layout) is obtained following a completely unattended scheme. External action is only required in the first step, to provide the filter specifications, which are the input variables of the proposed two-step ASM algorithm.

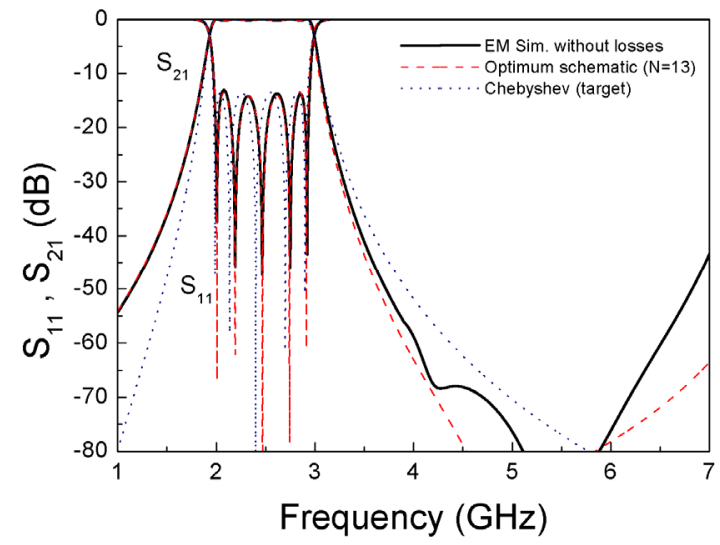

Fig. 8. Lossless electromagnetic simulation of the synthesized order-5 filter, compared to the response of the optimum filter schematic and target response.

(a)

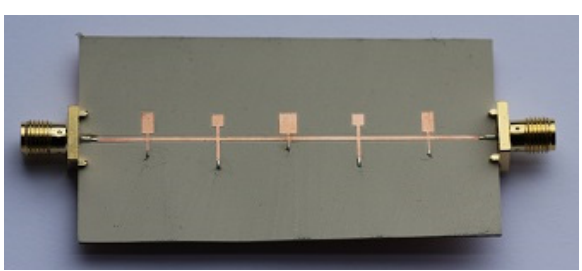




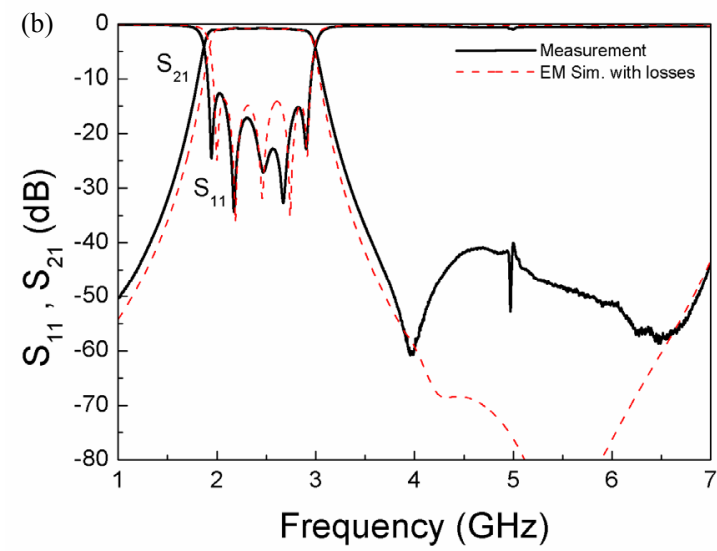

Fig. 9. Photograph of the fabricated order-5 filter (a) and measured response compared to the lossy electromagnetic simulation (b). The layout of the fabricated filter is the one depicted in Fig. 2.

At this point it is worth mentioning that the scheme of the second ASM is similar to the so-called knowledge embedded space mapping technique reported in [16], in the sense that we make use of analytical formulas to link dimensions (layout geometry) and parameters of equivalent circuit model (inductances and capacitances for the resonators). However, there is a noticeable difference with regard to the cited work, since in our case we avoid the need of using an embedded buffer space, and we have solved the problem of employing a Jacobian matrix B that can directly relate circuit elements with layout geometrical dimensions. Furthermore, the key point of the present paper is the first ASM scheme providing the optimum filter schematic.

On the other hand, for the optimization of filter layout (second ASM), an alternative solution could be port tuning ASM [17]. Nevertheless, it has been found that the proposed procedure, based on a classical ASM approach with three sub-processes, provides convergence in few steps and accurate results. The reason is that for the worst subprocess (resonator optimization), only three variables are involved, the initial layout is very close to the target and no significant inter-resonator coupling arises. Thus, we have opted to apply the proposed ASM scheme for the second ASM (layout generation).

\section{DESIGN OF A $9^{\mathrm{TH}}$-ORDER FILTER}

In order to demonstrate the potential of the proposed twostep ASM algorithm for the design and optimization of the considered type of filters, let us now apply the developed tool to the design of a higher order filter. In [9], a $7^{\text {th }}$-order filter (where the first ASM algorithm was not applied) was synthesized. Therefore, in this section we consider the design of a $9^{\text {th }}$-order filter. The specifications are: $f_{0}=1$ $\mathrm{GHz}, F B W=45.3 \%$ ( $-3-\mathrm{dB}$ fractional bandwidth) and $L_{A r}=$ $0.4 \mathrm{~dB}$. Application of the first ASM algorithm gives the schematic with element values indicated in table IV and $90^{\circ}$ (at $f_{0 \mathrm{f}}^{(7)}=1.0311 \mathrm{GHz}$ ) transmission line sections. Convergence has been achieved after $N=7$ iterations, when the error function is as small as $0.63 \%$.
TABLE IV

ELEMENT VALUES OF THE SHUNT RESONATORS FOR THE OPTIMUM FILTER SCHEMATIC OF THE $9^{\mathrm{TH}}$ ORDER FILTER

\begin{tabular}{|c|c|c|c|}
\hline Stage & $\boldsymbol{L}_{\boldsymbol{p}}(\mathrm{nH})$ & $\boldsymbol{L}_{\boldsymbol{r}}(\mathrm{nH})$ & $\boldsymbol{C}_{\boldsymbol{r}}(\mathrm{pF})$ \\
\hline 1,9 & 5.2439 & 1.7480 & 3.4077 \\
\hline 2,8 & 4.7471 & 1.5824 & 3.7643 \\
\hline 3,7 & 3.0439 & 1.0146 & 5.8707 \\
\hline 4,6 & 4.2765 & 1.4255 & 4.1786 \\
\hline 5 & 2.9547 & 0.9849 & 6.0478 \\
\hline
\end{tabular}

Application of the second ASM algorithm, considering the substrate used for the $5^{\text {th }}$-order filter (Rogers RO3010 with thickness $h=635 \mu \mathrm{m}$ and dielectric constant $\varepsilon_{r}=$ 10.2), provides the filter geometry indicated in Table V. For this filter, we have meandered the line sections in order to reduce device dimensions.

TABLE V

GEOMETRY PARAMETERS OF THE SYNTHESIZED ORDER-9 FILTER

\begin{tabular}{|c|c|c|c|c|c|c|}
\hline Stage & $\begin{array}{c}\boldsymbol{l}_{\boldsymbol{L} \boldsymbol{r}} \\
(\mathrm{mm})\end{array}$ & $\begin{array}{c}\boldsymbol{l}_{\boldsymbol{C} \boldsymbol{r}} \\
(\mathrm{mm})\end{array}$ & $\begin{array}{c}\boldsymbol{l}_{\boldsymbol{L} \boldsymbol{p}} \\
(\mathrm{mm})\end{array}$ & $\begin{array}{c}\boldsymbol{l}_{\text {cell }} \\
(\mathrm{mm})\end{array}$ & $\begin{array}{c}\boldsymbol{W}_{\boldsymbol{C} \boldsymbol{r}} \\
(\mathrm{mm})\end{array}$ & $\begin{array}{c}\boldsymbol{W}_{\boldsymbol{L p}} \\
(\mathrm{mm})\end{array}$ \\
\hline 1,9 & 4.6273 & 4.0264 & 10.5715 & 27.5994 & 3 & 0.5 \\
\hline 2,8 & 4.0219 & 3.6428 & 9.6523 & 27.5952 & 4 & 0.5 \\
\hline 3,7 & 2.1062 & 5.4739 & 6.2752 & 27.5922 & 5 & 0.5 \\
\hline 4,6 & 3.3853 & 4.344 & 8.7157 & 27.6578 & 4 & 0.5 \\
\hline 5 & 2.0049 & 5.6627 & 6.1136 & 27.5662 & 5 & 0.5 \\
\hline
\end{tabular}

Figure 10 shows the layout of the filter and the lossless electromagnetic simulation, compared to the optimum filter schematic and target responses. The fabricated filter is depicted in Fig. 11, together with the measured response and the lossy electromagnetic simulation. Again, very good agreement between the different responses can be appreciated. Moreover, the filter response satisfies the considered specifications. Thus, it has been demonstrated that the proposed and developed two-step ASM tool is able to automatically provide the layout of the considered type of filters for different orders and specifications.

(a)

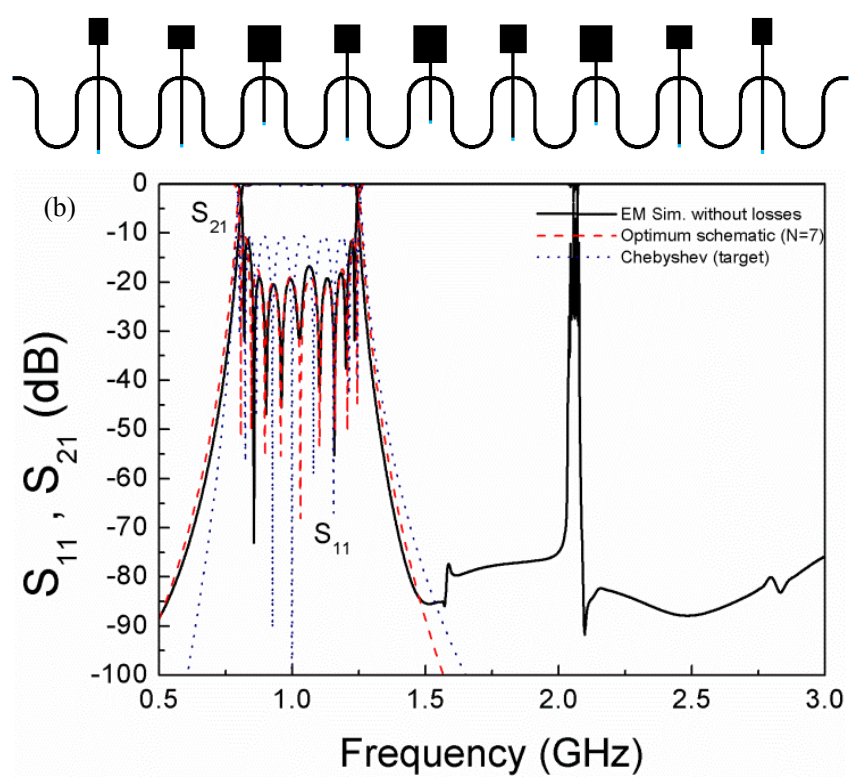

Fig. 10. Layout of the synthesized order-9 filter (a), and lossless electromagnetic simulation compared to the response of the optimum filter schematic and target response (b). 


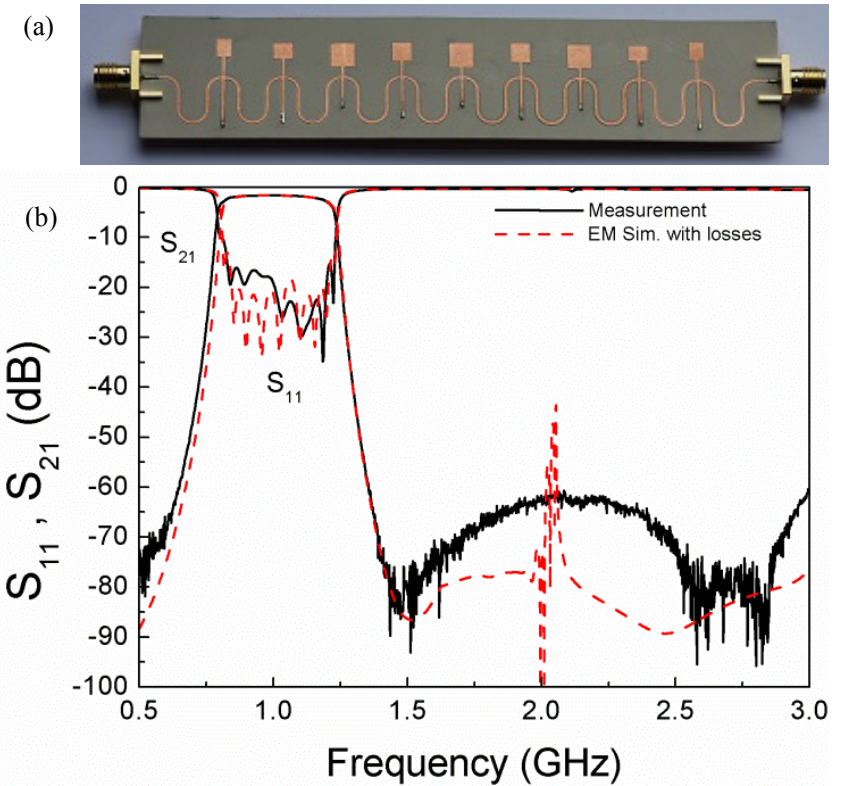

Fig. 11. Photograph of the fabricated order-9 filter (a) and measured response compared to the lossy electromagnetic simulation (b).

\section{CONCLUSIONS}

In conclusion, we have developed an iterative tool for the automatic optimization of bandpass filters based on a combination of stepped impedance resonators (SIRs) and shunt inductive stubs coupled through admittance inverters. These filters are useful to simultaneously achieve wide fractional bandwidths, high selectivity and spurious-free responses up to high frequencies (thanks to the inherent transmission zeros provided by the SIRs). The tool is based on a two-step aggressive space mapping (ASM) algorithm, where the optimum filter schematic is first determined from the filter specifications. Once the filter schematic is inferred, the element values are the inputs of the second ASM algorithm, which provides the final filter layout, determining the free geometrical parameters of each filter cell independently. The main relevant contribution of this paper is the development of the first ASM algorithm, able to provide a filter schematic (optimum) fully satisfying the specifications. In this optimum schematic the admittance inverters are implemented by means of transmission line sections exhibiting an electrical length of $90^{\circ}$ at a frequency provided by the ASM algorithm (this frequency does not necessarily coincide with the filter central frequency). Indeed, in the first ASM algorithm, the set of specifications are considered to be the variables in both the optimization and validation spaces, and the iterative ASM process ends when the variables of the optimization space coincide with the target (specifications). The developed two-step ASM tool has been proven with the design of a $5^{\text {th }}$-order filter, which has been used as an example to conduct and give the details of the tool, and then it has been successfully applied to the complete design of a $9^{\text {th }}$-order filter. In both cases, it has been found that the response of the optimum filter schematic (generated by the first ASM) satisfies the specifications, and that the generated layout (second ASM) exhibits a frequency response in very good agreement with that of the schematic. Thus, the developed two-step ASM design technique has been validated by means of two different examples, including a very high order filter. In both examples, the layout of the filters has been inferred in a completely unattended optimization scheme.

\section{REFERENCES}

[1] J. W. Bandler, R. M. Biernacki, S. H. Chen, P. A. Grobelny and R. H. Hemmers, "Space mapping technique for electromagnetic optimization", IEEE Trans. Microw. Theory Techn., vol. 42, pp. 2536-2544, Dec. 1994.

[2] J. W. Bandler, R. M. Biernacki, S. H. Chen, R. H. Hemmers, and K. Madsen, "Electromagnetic optimization exploiting aggressive space mapping", IEEE Trans. Microw. Theory Techn., vol. 43, pp. 2874 2882, Dec. 1995.

[3] M. H. Bakr, J. W. Bandler, K. Madsen, J. E. Rayas-Sánchez and J. Søndergaard, "Space-mapping optimization of microwave circuits exploiting surrogate models", IEEE Trans. Microw. Theory Techn., vol. 43, pp. 2297-2306, Dec. 2000.

[4] S. Koziel, Q. S. Cheng, and J. W. Bandler, "Space mapping", IEEE Microwave Magazine, vol. 9, pp. 105-122, Dec. 2008.

[5] J. W. Bandler, R. M. Biernacki, S. H. Chen, and D. Omeragic, "Space mapping optimization of waveguide filters using finite element and mode-matching electromagnetic simulators", IEEE MTTS Int. Microwave Symp., June 1997, Denver, CO (USA).

[6] J. W. Bandler, Q. S. Cheng, D. M. Hailu, and N. K. Nikolova, "A space-mapping design framework", IEEE Trans. Microw. Theory Techn., vol. 53, pp. 2601-2610, Nov. 2004.

[7] J. V. Morro, P. Soto, H. Esteban, V. E. Boria, C. Bachiller, M. Taroncher, S. Cogollos and B. Gimeno, "Fast automated design of waveguide filters using aggressive space mapping with a new segmentation strategy and a hybrid optimization algorithm", IEEE Trans. Microw. Theory Techn., vol. 53, pp. 1130-1142, April 2005.

[8] J. Bonache, I. Gil, J. García-García and F. Martín, "Compact microstrip band-pass filters based on semi-lumped resonators", IET Microwaves Antennas and Propagation, vol. 1, pp. 932-936, August 2007.

[9] J. Selga, M. Sans, A. Rodríguez, J. Bonache, V. Boria, and F. Martín, "automated synthesis of planar wideband bandpass filters based on stepped impedance resonators (SIRs) and shunt stubs through aggressive space mapping (ASM)", IEEE MTT-S Int. Microwave Symp., June 2014, Tampa, FL (USA).

[10] J. W. Bandler, Q. S. Cheng, S. A. Dakroury, A. S. Mohamed, M. H. Bakr, K. Madsen, and J. Søndergaard, "Space mapping: the state of the art," IEEE Trans. Microw. Theory Techn., vol. 52, pp. 337-361, 2004.

[11] J. S. Hong and M. J. Lancaster, Microstrip Filters for RF/Microwave Applications, John Wiley \& Sonc Inc., 2001.

[12] MATLAB ver. 2010a, The MathWorks Inc., 2010.

[13] Agilent Momentum ver. 8.20.374, Agilent Technologies, 2008.

[14] D. M. Pozar, Microwave Engineering, Addison Wesley, 1990.

[15] I. Bahl and P. Barthia, Microwave Solid State Circuit Design, John Wiley, 1998.

[16] K. L. Wu, R. Zhang, M. Ehlert, and D. G. Fang, "An explicit knowledge-embedded space mapping technique and its application to optimization of LTCC RF passive circuits," IEEE Trans. Compon. Packaging Techn., vol. 26, pp. 399-406, June 2003.

[17] Q. S. Cheng, J. C. Rautio, J. W. Bandler, and S. Koziel, "Progress in simulator-based tuning-the art of tuning space mapping," IEEE Microw. Mag., vol. 11, pp. 96-110, June 2010.

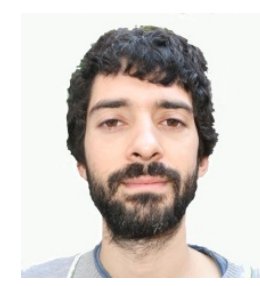

Marc Sans was born in Terrassa (Barcelona), Spain, in 1982. He received the B.S. Degree in Telecommunications Engineering - Electronic Systems in 2006, the M.S. Degree in Telecommunications Engineering in 2008 and the M.S. Degree in Electronics Engineering in 2013 from the Universitat Autònoma de Barcelona (UAB). In 2008 he started his professional career as a RF Engineer at Sony-FTVE developing the RF stage of TV receivers. In 2010 he moved to Mier Comunicaciones S.A. to carry out the design of passive and active devices for VHF-UHF broadcasting units. Since 2014 he is working towards the PhD Degree at CIMITEC-UAB in the synthesis of microwave devices based on EM optimization techniques.

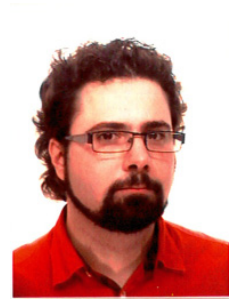

Jordi Selga (S'11-M'14) was born in Barcelona, Spain, in 1982. He received the B.S. Degree in Telecommunications Engineering - Electronic Systems in 2006, the M.S. Degree in Electronics Engineering in 2008 and the $\mathrm{PhD}$ degree in Electronics Engineering in 2013 from the Universitat Autònoma de Barcelona (UAB), Barcelona, Spain. Since 2008 is member of CIMITEC-UAB, a research centre on Metamaterials supported by TECNIO 
(Catalan Government). He was holder of a national research fellowship from the Formación de Profesorado Universitario Program of the Education and Science Ministry (Reference AP2008-4707). He is currently working in subjects related to metamaterials, CAD design of microwave devices, EM optimization methods and automated synthesis of planar microwave components at the UAB.

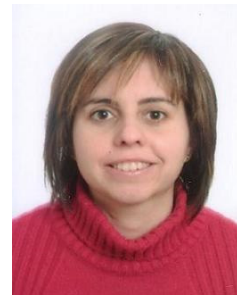

Ana Rodriguez (S'10) was born in Lugo, Spain. She received the Telecommunications Engineering degree from the Universidade de Vigo (UV), Spain, in 2008. As a student, she participated in the Erasmus exchange program, developing the Master Thesis at the University of Oulu, Finland. Since the end of 2008, she has joined the Institute of Telecommunications and Multimedia Applications (iTEAM), which is part of the scientific park at the Universitat Politècnica de València (UPV), Spain. She obtained "Master en Tecnología, Sistemas y Redes de Comunicaciones" in 2010 from UPV, and currently is on the way of pursuing Ph.D. degree. Her main research interests include CAD design of microwave devices, EM optimization methods and metamaterials.

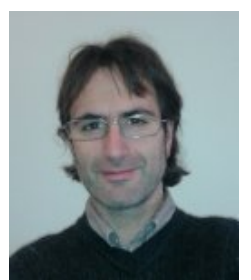

Jordi Bonache (S'05-M'07) was born in 1976 in Barcelona (Spain). He received the Physics and Electronics Engineering Degrees from the Universitat Autònoma de Barcelona in 1999 and 2001, respectively and the $\mathrm{PhD}$ degree in Electronics Engineering from the same university in 2007. In 2000, he joined the "High Energy Physics Institute" of Barcelona (IFAE), where he was involved in the design and implementation of the control and monitoring system of the MAGIC telescope. In 2001, he joined the Department of Electronics Engineering of the Universitat Autònoma de Barcelona where he is currently Lecturer. From 2006 to 2009 worked as executive manager of CIMITEC. Currently is leading the research in RFID and antennas in CIMITEC. His research interests include active and passive microwave devices, metamaterials, antennas and RFID.

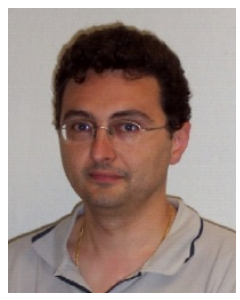

Vicente E. Boria (S'91-A'99-SM'02) was born in Valencia, Spain, on May 18, 1970. He received his "Ingeniero de Telecomunicación" degree (with first-class honors) and the "Doctor Ingeniero de Telecomunicación" degree from the Universidad Politécnica de Valencia, Valencia, Spain, in 1993 and 1997, respectively. In 1993 he joined the "Departamento de Comunicaciones", Universidad Politécnica de Valencia, where he has been Full Professor since 2003. In 1995 and 1996, he was holding a Spanish Trainee position with the European Space Research and Technology Centre, European Space Agency (ESTEC-ESA), Noordwijk, The Netherlands, where he was involved in the area of EM analysis and design of passive waveguide devices. He has authored or co-authored 7 chapters in technical textbooks, 75 papers in refereed international technical journals, and over 150 papers in international conference proceedings. His current research interests are focused on the analysis and automated design of passive components, left-handed and periodic structures, as well as on the simulation and measurement of power effects in passive waveguide systems.

Dr. Boria has been a member of the IEEE Microwave Theory and Techniques Society (IEEE MTT-S) and the IEEE Antennas and Propagation Society (IEEE AP-S) since 1992. He is reviewer of the IEEE Transactions on Microwave Theory and Techniques, Proceeding of the IET (Microwaves, Antennas and Propagation) and IET Electronics Letters. Since 2013, he serves as Associate Editor of IEEE Microwave and Wireless Components Letters. He is also a member of the Technical Committees of the IEEE-MTT International Microwave Symposium and of the European Microwave Conference.

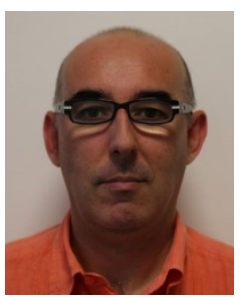

Ferran Martín (M'04-SM'08-F'12) was born in Barakaldo (Vizcaya), Spain in 1965 . He received the B.S. Degree in Physics from the Universitat Autònoma de Barcelona (UAB) in 1988 and the $\mathrm{PhD}$ degree in 1992. From 1994 up to 2006 he was Associate Professor in Electronics at the Departament d'Enginyeria Electrònica (Universitat Autònoma de Barcelona), and since 2007 he is Ful Professor of Electronics. In recent years, he has been involved in different research activities including modelling and simulation of electron devices for high frequency applications, millimeter wave and $\mathrm{THz}$ generation systems, and the application of electromagnetic bandgaps to microwave and millimeter wave circuits. He is now very active in the field of metamaterials and their application to the miniaturization and optimization of microwave circuits and antennas. He is the head of the Microwave Engineering, Metamaterials and Antennas Group (GEMMA Group) at UAB, and director of CIMITEC, a research Center on Metamaterials supported by TECNIO (Generalitat de Catalunya). He has organized several international events related to metamaterials, including Workshops at the IEEE International Microwave Symposium (years 2005 and 2007) and European Microwave Conference (2009), and the Fifth International Congress on Advanced Electromagnetic Materials in Microwaves and Optics (Metamaterials 2011), where he has acted as chair of the Local Organizing Committee. He has acted as Guest Editor for three Special Issues on Metamaterials in three International Journals. He has authored and co-authored over 450 technical conference, letter, journal papers and book chapters, he is co-author of the book on Metamaterials entitled Metamaterials with Negative Parameters: Theory, Design and Microwave Applications (John Wiley \& Sons Inc.), and he has generated 14 PhDs. Ferran Martín has filed several patents on metamaterials and has headed several Development Contracts.

Prof. Martín is a member of the IEEE Microwave Theory and Techniques Society (IEEE MTT-S). He is reviewer of the IEEE Transactions on Microwave Theory and Techniques and IEEE Microwave and Wireless Components Letters, among many other journals, and he serves as member of the Editorial Board of IET Microwaves, Antennas and Propagation and International Journal of RF and Microwave Computer-Aided Engineering. $\mathrm{He}$ is also a member of the Technical Committees of the European Microwave Conference (EuMC) and International Congress on Advanced Electromagnetic Materials in Microwaves and Optics (Metamaterials). Among his distinctions, Ferran Martín has received the 2006 Duran Farell Prize for Technological Research, he holds the Parc de Recerca UAB Santander Technology Transfer Chair, and he has been the recipient of two ICREA ACADEMIA Awards (calls 2008 and 2013). 